\title{
Metastatic Malignant Paraganglioma: A Case Report and Review of Literature
}

\author{
Carlo Angelo Prades ${ }^{\mathrm{a}, \mathrm{d}}$, Bassel Atassia, b, Hamid Nazeer ${ }^{\mathrm{c}}$
}

\begin{abstract}
Metastasis is a rare presentation of non-secretory paraganglioma. Consequently, there is no standard of care for the treatment of metastatic malignant paraganglioma. The most widely used chemotherapy regimen for non-resectable cases includes cyclophosphamide, vincristine, and dacarbazine (CVD). CVD has been previously studied with variable therapeutic response. However, yttrium-90 (Y90) radioembolization has not been previously studied in the treatment of hepatic metastasis in patients diagnosed with malignant paraganglioma. This case report follows the treatment of a patient with stage IV mediastinal paraganglioma with metastasis to the liver. Treatment consisted of the CVD chemotherapy regimen and Y 90 radioembolization of the hepatic lesions. After 10 cycles of CVD, the tumor size has decreased from $6.0 \times 8.8$ to $5.5 \times 3.0 \times 3.4 \mathrm{~cm}$ on computed tomography scan. The prominent metastatic liver lesions responded after Y90 radioembolization of both the right and left hepatic arteries. The prominent right hepatic lobe lesion has decreased in size from $2.6 \times 3.4 \times 3.0$ $\mathrm{cm}$ to $2.6 \times 2.8 \times 2.9 \mathrm{~cm}$. The prominent left hepatic lobe lesion originally measuring $1.6 \mathrm{~cm}$ in diameter completely resolved on follow-up imaging studies. After completion of 12 cycles of chemotherapy, the most recent positron emission testing scan determined no evidence of disease regarding both the primary mass and the hepatic lesions. This study demonstrates the first case of combination chemotherapy and Y90 radioembolization with a complete response per response evaluation criteria in solid tumors criteria. The approaches toward diagnosis and treatment corresponding to this case of malignant metastatic paraganglioma are also reviewed in this study.
\end{abstract}

Keywords: Paraganglioma; Metastatic paraganglioma; Malignant paraganglioma; Ytrrium-90 radioembolization

\section{Introduction}

Paragangliomas are rare neuroendocrine tumors of the auto-

\section{Manuscript accepted for publication May 17, 2017}

${ }^{a}$ Chicago Medical School, Woodridge, IL, USA

${ }^{\mathrm{b}}$ Cancer Center, Little Company of Mary Hospital, Evergreen Park, IL, USA

'Little Company of Mary Hospital, Evergreen Park, IL, USA

${ }^{\mathrm{d} C o r r e s p o n d i n g ~ A u t h o r: ~ C a r l o ~ A n g e l o ~ P r a d e s, ~ C h i c a g o ~ M e d i c a l ~ S c h o o l, ~} 6430$

Winston Dr., Woodridge, IL 60517, USA. Email: carlo.prades@my.rfums.org

doi: https://doi.org/10.14740/wjon1033w nomic ganglia with the ability to secrete catecholamines. The exact incidence of paragangliomas has not been established due to a similar clinical presentation shared with intra-adrenal autonomic tumors termed pheochromocytomas. The estimated combined prevalence is between 1:6,500 and 1:2,500, and the annual combined incidence is 500 - 1,600 cases of pheochromocytoma and paraganglioma [1]. Paragangliomas can have a benign or a malignant course. The incidence of malignant paraganglioma is rare and is estimated to be 93 per 400 million people [2]. The majority of paragangliomas are sporadic, but certain hereditary syndromes such as multiple endocrine neoplasia, neurofibromatosis, and von Hippel-Lindau syndrome increase the genetic predisposition to developing paragangliomas.

Paragangliomas can arise from any anatomical location with chromaffin-containing autonomic tissue from the base of the skull to the pelvis. Paragangliomas have been previously described in the head and neck regions, jugulotympanic area, bladder, and other locations leading to varying clinical presentations with the majority of cases commonly remaining asymptomatic [3]. We report a patient with metastatic malignant paraganglioma and review the literature involving the clinical presentation, diagnosis, and management of this disease leading to complete remission of the primary tumor and all metastatic lesions to the liver.

\section{Case Report}

A 51-year-old African-American male with no previous medical history presented to the clinic with progressive dysphagia to both solids and liquids. He denied both a history of smoking and a family history of medical conditions. Review of systems revealed severe back pain and declining daily performance status. He did not endorse any secretory symptoms such as paroxysmal headaches, diarrhea, or palpitations. Physical examination demonstrated no abnormal findings.

Initial imaging included computed tomography (CT) with oral and intravenous (IV) contrast of the chest which illustrated a posterior mediastinal soft tissue mass with necrosis measuring $6.0 \times 8.8 \mathrm{~cm}$ abutting but not encasing the distal thoracic esophagus (Fig. 1a) [4]. Two prominent hypodense lesions were localized in the right posterior hepatic lobe measuring $2.6 \times 2.8 \times 2.9 \mathrm{~cm}$ and the medial segment of the left hepatic lobe measuring $1.6 \mathrm{~cm}$ in diameter (Fig. 2a) [4].

Endoscopic ultrasound-directed needle biopsy results for the posterior mediastinal mass described a hemangiopericy- 

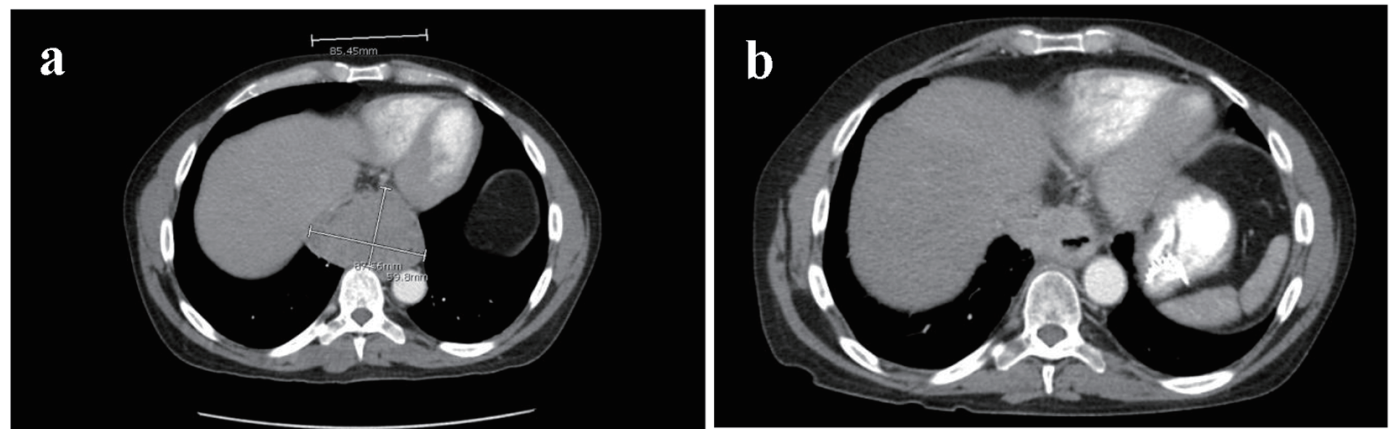

Figure 1. (a) Sagittal section of CT thorax with oral and IV contrast measuring posterior mediastinal mass prior to chemoradiation with dimensions of $6.0 \times 8.8 \mathrm{~cm}$. (b) Sagittal section of CT thorax with oral and IV contrast of posterior mediastinal mass after 10 cycles of CVD. Tumor size decreased to $5.5 \times 3.0 \times 4.0 \mathrm{~cm}$.

toma vascular pattern without defined nests. Immunostaining for synaptophysin was strong and diffuse. All other immunostains including pan-keratin, CD45, S-100, Kit, CAM5.2, desmin, CD34, smooth muscle actin, SOX10, melan A, and inhibin were negative. Ultrasound-directed core needle biopsy of the hepatic lesions described tumor growth in broad sheet and nests. Immunostaining was strongly positive for synaptophysin but negative for CAM5.2, S-100, chromogranin, CDx2, and T1F1. Ki-67 proliferative rate was noted at 50\%. The histological analysis combined with structural imaging diagnosed the patient with malignant posterior mediastinal paraganglioma with liver metastasis. Laboratory investigations included 24-h fractionated urinary catecholamines and metanephrines supporting a non-functional tumor (Table 1).

The mass was unamenable to surgical resection. The patient was stated on a chemotherapeutic regimen of cyclophosphamide $\left(750 \mathrm{mg} / \mathrm{m}^{2}\right.$ on day 1$)$, vincristine $\left(1.3 \mathrm{mg} / \mathrm{m}^{2}\right.$ on day $1)$, and dacarbazine $\left(600 \mathrm{mg} / \mathrm{m}^{2}\right.$ on day 1 and day 2$)$ every 3 weeks (CVD regimen). After eight cycles of chemotherapy, the patient underwent yttrium-90 (Y90) radioembolization of the prominent hepatic metastatic lesions. The right lobar dose was 1.35 gigabecquerel (GBq) in milliCurie (mCi) Y90 SIRSpheres microspheres with a total of 32.1 milligray (mGy) dose. The left lobar dose was $0.9 \mathrm{GBq}$ in mCi Y90 SIR-Spheres microspheres (Sirtex Medical, Sydney, Australia) with a total of $34.8 \mathrm{mGy}$ dose.

The patient completed 10 cycles of chemotherapy and experienced a gradual improvement in his back pain, weight, appetite, swallowing, and performance status throughout treatment. Follow-up CT scans after the 10th cycle showed near complete response of the tumor and a decrease in size of the hepatic lesions. CT scan with IV and oral contrast measured the mediastinal mass to be $5.5 \times 3.0 \times 4.0 \mathrm{~cm}$ (Fig. 1b) [5]. The posterior right hepatic lobe lesion decreased in size to $2.6 \times 2.8$ $\times 2.9 \mathrm{~cm}$, and the left hepatic lobe lesion completely resolved (Fig. 2b) [5]. These interval decreases in the hepatic metastasis corresponded to a partial response per response evaluation criteria in solid tumors (RECIST) criteria [6]. The patient finished a total of 12 cycles of chemotherapy with subsequent resolution of the primary mass and the hepatic lesions, thus corresponding with a complete response per RECIST criteria.

\section{Discussion}

The clinical presentation of paragangliomas varies with tumor location, degree of tumor burden, and secretory functionality. Functional paragangliomas secrete excessive catecholamines
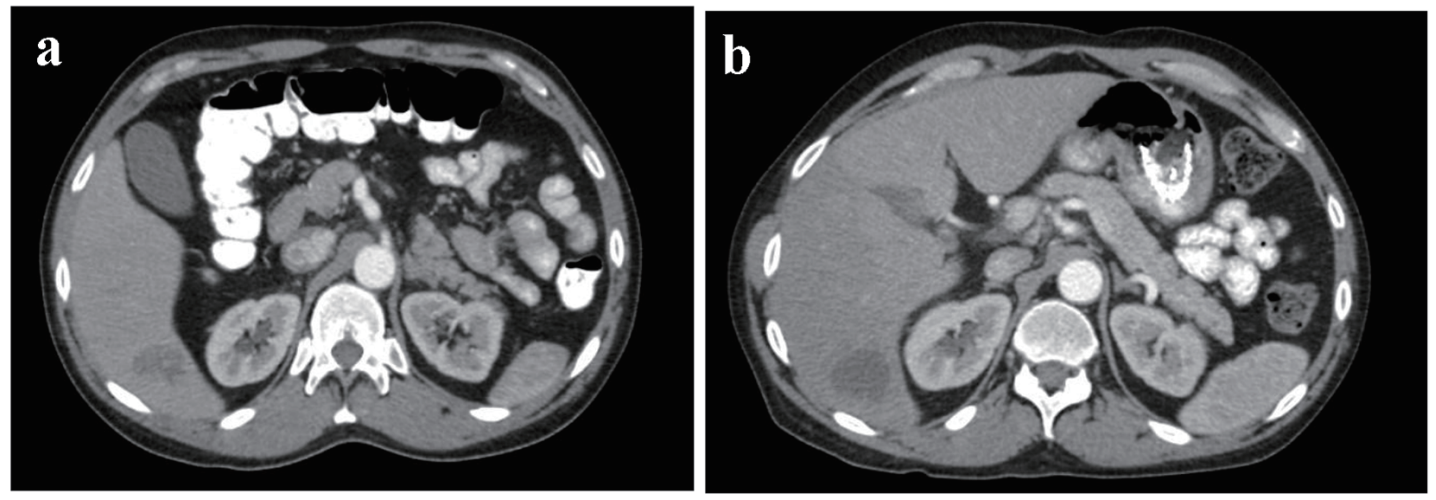

Figure 2. (a) Sagittal section of CT thorax with oral and IV contrast demonstrating posterior right hepatic lobe mass $(2.6 \times 3.4 \times$ $3.0 \mathrm{~cm}$ ) prior to starting chemoradiation. (b) Sagittal section of CT thorax with oral contrast demonstrating posterior right hepatic lobe measuring $2.6 \times 2.8 \times 2.9 \mathrm{~cm}$ after 10 cycles of CVD regimen and trans-arterial Y90 radioembolization of right and left hepatic arteries. 
Table 1. Fractionated Urinary Catecholamines and Reference Ranges

\begin{tabular}{lll}
\hline & $\begin{array}{l}\text { Actual value } \\
(\boldsymbol{\mu g} / \mathbf{2 4} \mathbf{~ h})\end{array}$ & $\begin{array}{l}\text { Reference range } \\
(\boldsymbol{\mu g} / \mathbf{2 4} \mathbf{~ h})\end{array}$ \\
\hline Epinephrine (EPI) & 20 & $2-24$ \\
Norepinephrine (NE) & 144 & $26-121$ \\
Total EPI + NE & 164 & $26-121$ \\
Dopamine & 418 & $52-480$ \\
Metanephrines & 239 & $90-315$ \\
Normetanephrines & 611 & $122-676$ \\
Total Metanephrines & 850 & $224-832$ \\
\hline
\end{tabular}

resulting in a clinical presentation of paroxysmal hypertension, headache, sweating, and palpitations [3, 7]. Non-functional paragangliomas typically present with symptoms such as dysphagia, angina, or dyspnea as a result of a compressive mass effect on adjacent structures [3, 7]. Mediastinal paragangliomas are estimated to make up $0.3 \%$ of all mediastinal tumors and less than $2 \%$ of all paragangliomas [8]. To date, the only reliable criterion for malignancy is the presence of metastatic spread of chromaffin cells in tissues that normally do not contain such cells [9].

Laboratory testing includes 24-h urine collection and measurement of urinary fractionated metanephrines and catecholamines. Testing should be done for patients with no symptoms related to excessive catecholamine secretion. The sensitivity of this urine collection study has been reported to be $84 \%$ for norepinephrine, $74 \%$ total metanephrines, $18 \%$ dopamine, and 14\% epinephrine, respectively [2]. However, Chen et al reported greater sensitivities of $97 \%$ for urinary metanephrines and $86 \%$ for urinary catecholamines [1]. Histological studies are required for definitive diagnosis. Previous studies described a Zellballen appearance in which chief cell nests are surrounded by sustentacular supporting cells [9]. Tumor markers include neuron-specific enolase (92.1\% sensitivity), chromogranin (84.2\%), and met-enkephalin (73\%) [10]. Histological studies have also denoted a negative keratin marker and the appearance of S-100 and glial fibrillary acid protein (GFAP) in the surrounding sustentacular cells $[9,10]$.

The standard of care for cases of early stage mediastinal paraganglioma is complete surgical resection. Currently, there are no standardized treatment guidelines for malignant mediastinal paraganglioma. For cases of metastatic disease, systemic therapy may be employed to decrease tumor burden. Averbuch et al demonstrated the efficacy of combination chemotherapy with $750 \mathrm{mg} / \mathrm{m}^{2}$ cyclophosphamide, $1.4 \mathrm{mg} /$ $\mathrm{m}^{2}$ vincristine, and $600 \mathrm{mg} / \mathrm{m}^{2}$ dacarbazine in the treatment of malignant paraganglioma [11]. A long-term follow-up study of 18 patients recommended the use of the CVD regimen in patients with malignant paraganglioma or pheochromocytoma resulting in $11 \%(2 / 18)$ complete response rate and $44 \%(8 / 18)$ partial response rate [12]. Although many studies have demonstrated partial remissions and complete remissions in some cases, there are no data of chemotherapy regimens prolonging survivalin cases of metastatic disease [11-14].
Liver-directed radioembolization of Y90 microsphere resins is used for local radiation exposure using the altered arterial network supplying the tumor. This local irradiation allows for a concentrated emission of target blood vessels while maintaining tolerable toxicity levels in the surrounding parenchyma [15]. Y90 radioembolization has been described as an emerging form of in situ cytoreductive treatment of hepatic metastasis of neuroendocrine tumors with variable response rates $[16$, 17].

To our knowledge, this is the first reported case of combination CVD therapy and Y90 radioembolization for metastatic malignant paraganglioma. Our patient thus far has demonstrated remarkable clinical and radiological improvement with 10 cycles of the CVD regimen. Follow-up CT scans demonstrated a progressive decrease in tumor size throughout chemotherapy cycles. The decrease in size of the posterior mediastinal mass is likely attributed to a similar chemotherapy schedule as demonstrated previously in prior studies $[9,10]$. The patient has demonstrated a decrease in the dominant metastatic liver lesions shortly after radioembolization of both the right and left hepatic arteries (Fig. 2a, b). The RECIST criteria monitor the imaging response to treatment of solid tumors. By applying this model's calculation to this case, we have calculated a $58 \%$ decrease in the patient's metastatic liver lesions. This corresponds with a complete response per RECIST criteria [6]. The most recent imaging studies after 12 cycles of chemotherapy and localized radiation therapy with Y90 radioembolization have shown complete remission of the primary posterior mediastinal tumor as well as all hepatic lesions to date.

\section{Conclusion}

Metastatic malignant non-secretory paraganglioma is an extremely rare disease. There have been few studies examining the use of combination chemotherapy and Y90 radioembolization for the treatment of malignant metastatic paraganglioma. To our knowledge, this case demonstrates the first study in which a patient with malignant paraganglioma with metastasis to the liver achieved a complete response as a result of the CVD regimen and Y90 radioembolization. In summary, the CVD regimen has shown to have therapeutic efficacy in treating paraganglioma consistent with the results of previous studies. This study also demonstrates the therapeutic utility of Y90 radioembolization of liver metastasis in cases of malignant paraganglioma. Future randomized clinical trials are required to support the therapeutic findings of combination chemotherapy and Y90 radioembolization demonstrated in this case.

\section{Financial Disclosures}

None.

\section{Conflicts of Interest}

All authors report no conflicts of interest in the preparation of 
this manuscript.

\section{References}

1. Chen H, Sippel RS, O'Dorisio MS, Vinik AI, Lloyd RV, Pacak K. The North American Neuroendocrine Tumor Society consensus guideline for the diagnosis and management of neuroendocrine tumors: pheochromocytoma, paraganglioma, and medullary thyroid cancer. Pancreas. 2010;39(6):775-783.

2. Welander J, Soderkvist P, Gimm O. Genetics and clinical characteristics of hereditary pheochromocytomas and paragangliomas. Endocr Relat Cancer. 2011;18(6):R253276.

3. Erickson D, Kudva YC, Ebersold MJ, Thompson GB, Grant CS, van Heerden JA, Young WF, Jr. Benign paragangliomas: clinical presentation and treatment outcomes in 236 patients. J Clin Endocrinol Metab. 2001;86(11):5210-5216.

4. Nazeer H. Initial CT Scan. Evergreen Park, IL: Little Company of Mary. 2016, Jan.

5. Nazeer H. CT Scan after 10 cycles. Evergreen Park, IL: Little Company of Mary. 2017, Feb.

6. Therasse P, Arbuck SG, Eisenhauer EA, Wanders J, Kaplan RS, Rubinstein L, Verweij J, et al. New guidelines to evaluate the response to treatment in solid tumors. European Organization for Research and Treatment of Cancer, National Cancer Institute of the United States, National Cancer Institute of Canada. J Natl Cancer Inst. 2000;92(3):205-216.

7. Gonzalez-Santos JM, Arnaiz-Garcia ME, Munoz-Herrera A, Lopez-Rodriguez J. Mediastinal paraganglioma fed by the left circumflex artery. Interact Cardiovasc Thorac Surg. 2016;23(5):835-836.

8. Wald O, Shapira OM, Murar A, Izhar U. Paraganglioma of the mediastinum: challenges in diagnosis and surgical management. J Cardiothorac Surg. 2010;5:19.

9. Ayadi-Kaddour A, Braham E, Ismail O, Smati B, Djilani H, El Mezni F. Posterior mediastinal paragangliomas: a report of three patients with peculiar tumours. Respirology. 2009; 14(3):459-461.

10. Moran CA, Suster S, Fishback N, Koss MN. Mediastinal paragangliomas. A clinicopathologic and immunohistochemical study of 16 cases. Cancer. 1993;72(8):23582364.

11. Kliewer KE, Cochran AJ. A review of the histology, ultrastructure, immunohistology, and molecular biology of extra-adrenal paragangliomas. Arch Pathol Lab Med. 1989;113(11):1209-1218.

12. Averbuch SD, Steakley CS, Young RC, Gelmann EP, Goldstein DS, Stull R, Keiser HR. Malignant pheochromocytoma: effective treatment with a combination of cyclophosphamide, vincristine, and dacarbazine. Ann Intern Med. 1988;109(4):267-273.

13. Huang H, Abraham J, Hung E, Averbuch S, Merino M, Steinberg SM, Pacak K, et al. Treatment of malignant pheochromocytoma/paraganglioma with cyclophosphamide, vincristine, and dacarbazine: recommendation from a 22year follow-up of 18 patients. Cancer. 2008;113(8):20202028.

14. Nomura K, Kimura H, Shimizu S, Kodama H, Okamoto T, Obara T, Takano K. Survival of patients with metastatic malignant pheochromocytoma and efficacy of combined cyclophosphamide, vincristine, and dacarbazine chemotherapy. J Clin Endocrinol Metab. 2009;94(8):2850-2856.

15. Fishbein L, Bonner L, Torigian DA, Nathanson KL, Cohen DL, Pryma D, Cengel KA. External beam radiation therapy (EBRT) for patients with malignant pheochromocytoma and non-head and -neck paraganglioma: combination with 131I-MIBG. Horm Metab Res. 2012;44(5):405410.

16. Murthy R, Habbu A, Salem R. Trans-arterial hepatic radioembolisation of yttrium-90 microspheres. Biomed Imaging Interv J. 2006;2(3):e43.

17. Memon K, Lewandowski RJ, Mulcahy MF, Riaz A, Ryu RK, Sato KT, Gupta R, et al. Radioembolization for neuroendocrine liver metastases: safety, imaging, and long-term outcomes. Int J Radiat Oncol Biol Phys. 2012;83(3):887-894. 